\title{
Freihandelszone Shenzhen in der VR-China: Experiment im Rahmen der neuen Wirtschaftspolitik
}

\section{Einleitung}

Bis zum Jahre 1979, in welchem sich die chinesische Parteiführung für die Einrichtung von Special Economic Zones (SEZ) in Shenzhen, Zhuhai, Xiamen und Shantou entschloss, waren die Freihandelszonen (Export Processing Zones/EPZ) lediglich von mehr oder weniger marktwirtschaftlich ausgerichteten Entwicklungsländern her bekannt. Die erste Freihandelszone wurde 1962 in Puerto Rico eingerichtet, als zollpolitische Enklave sozusagen. Den in der EPZ niedergelassenen Unternehmungen wurde zollfreie Einfuhr ihrer Produktionsmittel bei gleichzeitig zollfreiem Export ihrer Fertigprodukte garantiert. Der EPZ in Puerto Rico folgte 1965 eine EPZ in Indien. Die achtziger Jahre schliesslich bedeuteten einen wahren Durchbruch, gab es doch 1981 bereits 24 EPZ in Ost- und Südostasien, $10 \mathrm{im}$ Nahen und Mittleren Osten, 4 in Afrika, 6 in der Karibik, 15 in Lateinamerika und eine im pazifischen Raum (WONG/CHU 1984). Zur Zeit scheinen jedoch insbesondere die ASEANLänder eher eine zurückhaltende EPZ-POlitik zu betreiben.

\section{Zielsetzungen}

Die eigentliche stossrichtung bei der Errichtung von Freihandelszonen in verschiedenen Entwicklungsländern liegt im beschleunigten Durchsetzen einer ganzen Palette von entwicklungspolitischen Zielsetzungen. Eine solche Wirtschaftspolitik weist folgende Elemente auf:

- Anziehung von Auslandinvestoren

- Import von technologischem know-how

- Erschliessung von Devisenquellen

- Schaffung moderner Produktionsstandorte

- Bereitstellung neuer Arbeitsplätze

- Einführung von modernem Management

- Stärkung der lokalen Infrastruktur: "Katalysatorwirkung"

- Bessere Nutzung der lokalen Rohstoffbasis
Eine Attraktivität ersten Ranges bedeutet für Auslandinvestoren vor allem die Möglichkeit, in den EPZ die zollschranken $z u$ umgehen. Allerdings genügt die zollfreiheit allein nicht, um neue Produktionsstandorte für fremde Unternehmungen $\mathrm{zu}$ schaffen. Es bedarf zusätzlicher Instrumentarien, mit welchen Entwicklungsländer ausländische Firmen zur Niederlassung in die jeweiligen Freihandelszonen bewegen können. Trotz einer grossen Vielfalt an solchen Instrumentarien, zeichnen sich bei allen EPZ gemeinsame Tendenzen ab:

- Keine Kontrolle des Devisenflusses

- Steuervergünstigungen

- Günstiger Land- und Immobilienerwerb

- Lange Pachtzeit

- Vorteilhafte Kreditbedingungen

- "Joint ventures" oder ausschliesslich fremder Kapitalbesitz

Dem jeweiligen Zonenland wird bei der Errichtung von EPZ meist eine ausserordentlich investitions-intensive Vorleistung abverlangt, um das Interesse des Auslandinvestors an kostenmässig und operationell günstigen standort- und Produktionsbedingungen aufrecht $\mathrm{zu}$ halten. Das Zonenland muss folgende strukturelle Vorteile anbieten können:

- Billige Arbeitskräfte

- Bereitstellung von Infrastruktur

- Bereitstellung von standorten für die Produktionseinrichtungen

- Zentralisierte und vereinheitlichte Administration

- Sicherung des "Arbeitsfriedens"
Dušan Šimko, Dr., Geographisches Institut der Universität Basel, Klingelbergstr. 16, 4056 Basel 


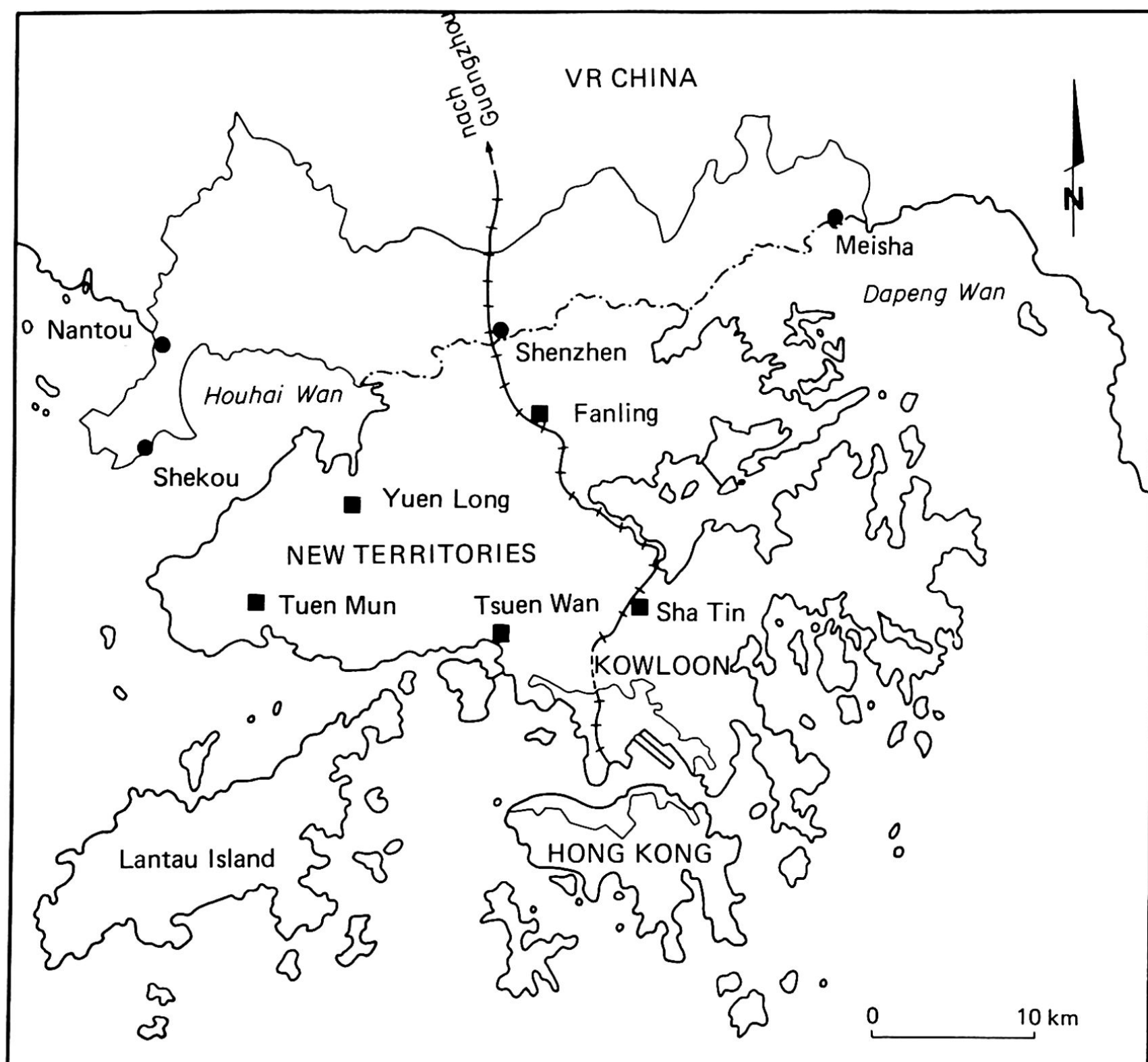

Entwurf: D. Šimko 1985

Abb. 1 Hongkong und Shenzhen SEZ

New Towns

- - - - Staatsgrenze

Hongkong - VR China

\section{Sozialpolitische Rahmenbedingungen in der VR-China}

Zwei Zäsuren markieren die grosse Wende in der ökonomischen Politik der Volksrepublik China: der Tod des Vorsitzenden Mao Zedong am 9. Sept. 1976 und die nachfolgende "Entmachtung der Viererbande" am 6. Oktober 1976. Damit gehörten die Wirtschaftsversuche im Rahmen der "Hundertblumenkampagne" (1956) und des "Grossen Sprunges Vorwärts" (1957) - die beide eine katastrophale Hungers- not bewirkt hatten - endgültig der Vergangenheit an. Um Liu Shaogi und Deng Xiaoping bildete sich ein Kreis von "rechten" Pragmatikern, der Maos primär sozial-utopisch geprägte Politik durch eine auf wirtschaftlicher Vernunft basierende Politik ersetzen wollte. Auf dem 3. Plenum des 11. Zentralkomitees der KP Chinas wurde die neue chinesische Wirtschaftsreform zum ersten Male der Parteispitze vorgestellt:

Freigabe der Lebensmittelpreise, Verlagerung von Wirtschaftsentscheidungs- 
kompetenzen auf niedrigere Ebenen der Parteihierarchie, begrenzte Entscheidungskompetenzen der sozialistischen Experimentalbetriebe mit der Möglichkeit, Planüberschüsse zurückzubehalten, Beschneidung der staatlichen Subventionen, verstärkte "Reprivatisierung" der kommunalen Dienstleistungsbetriebe, $\mathrm{Re}-$ form der Volkskommunen, Konstituierung von Freihandelszonen (SEZ) des "sozialistischen Typus". Dieser Massnahmekatalog der chinesischen Wirtschaftsreform wurde durch das Zentralkomitee der KP Chinas am 20. Oktober 1984 offiziell verankert. Die Reformbestrebungen scheinen vorläufig trotz einiger Rückschläge in ihrer Gesamtbilanz eher positiv auszufallen. Angesichts einer drohenden wirtschaftlichen Ueberhitzung hat die Regierung im letzten Jahr des 6. Fünfjahresplanes (1981 - 1985) auf langsame Gangart ungeschaltet (KOSTA 1985).

\section{Experimentalcharakter der SEZ}

Freimütig räumen die chinesischen Oekonomen ein, dass die vor sechs Jahren eingerichteten SEZ einen ausgesprochenen Experimentalcharakter haben (INTERNATIONAL HERALD TRIBUNE 1985). Es sind weitere 14 SEZ-Projekte an zusätzlichen Lokalitäten im küstenbereich und auf der Insel Hainan geplant (THE SOUTH CHINA MORNING POST 1985).

Gelegentlich wird das SEZ-Experiment der Volkschinesen mit der sowjetischen NEP-Politik (1921-1928) verglichen. Da$\mathrm{zu}$ ist $\mathrm{zu}$ bemerken, dass ein solcher Vergleich zumindest ungenau ist, da die heutige chinesische Abweichung von der "linken" Wirtschaftsutopie noch lange nicht die Zuwendung zum marktorientierten Sozialismus bedeutet.

Der eigentliche Spezialaspekt der chinesischen Freihandelszonen liegt in der Eigentumsfrage der Produktionsmittel und im besonderen Anpassungszwang an die neu kreierte Entscheidungsautonomie. Man darf dabei nicht vergessen, dass die Chinesen die Idee des Freihandelszonenmarktes lediglich übernommen und modifiziert, aber nicht entworfen haben.

\section{Shenzhen Special Economic Zone}

Der kommunale Verwaltungsbezirk Shenzhen $\left(22^{\circ} \mathrm{N}, 113^{\circ} \mathrm{E}\right)$ befindet sich im südlichen Teil der chinesischen Provinz Guangdong ( $\mathrm{Abb} 1$ ). Shenzhen schliesst im Süden an die New Territories von Hongkong an und grenzt an die Verwaltungsbezirke (xians) Dongguan (N) und Huiyang (S). Die westliche Grenze von
Shenzhen bildet die Deltaflanke von $\mathrm{zhu}$ Jiang Kou. Die Gesamtfläche von Shenzhen beträgt $2 \cdot 020 \mathrm{~km}^{2}$, und die Wohnbevölkerung beläuft sich auf ca. 320'000 Personen.

Ursprünglich war der Status einer SEZ für den ganzen kommunalen Verwaltungsbezirk Shenzhen geplant. Aber erst im September 1980 wurde die Grenzziehung von Shenzhen SEZ definitiv abgeschlossen. Die Freihandelszone mit dem Verwaltungszentrum Shenzhen/Shangbu erstreckt sich von der Freihafenenklave Shekou bis zur Stadt Meisha und misst $327,5 \mathrm{~km}^{2}$.

Für den Entscheid der Wirtschaftskommission des Zentralkomitees der KP Chinas, in Shenzhen eine SEZ einzurichten, sprachen offensichtlich folgende Beweggründe:

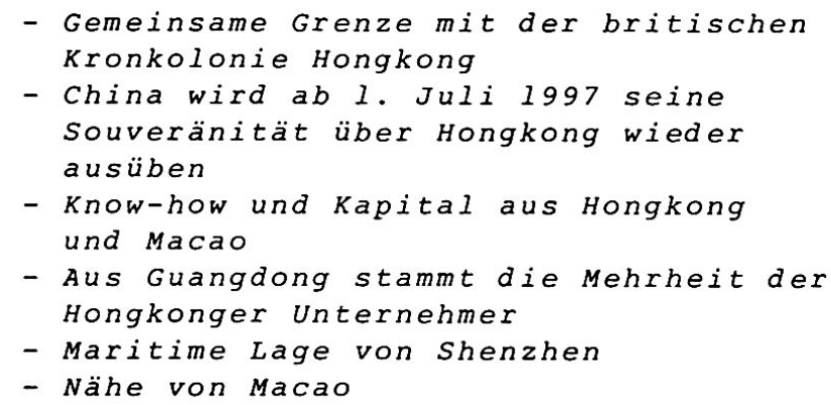

Shenzhen hat die Funktion des traditionellen Einfalltores von Südchina wahrgenommen. Bereits 1912 wurde - dank der Kowloon-Canton Railway - die Eisenbahnverbindung zwischen dem Finanzplatz Hongkong und der Provinzhauptstadt Kanton (Guangzhou) verwirklicht. Die Modernisierung der Eisenbahnlinie, die vor zwei Jahren erfolgreich abgeschlossen wurde, verkürzt die Zugreise von Hongkong nach Guangzhou auf drei Fahrstunden. Seit 1984 besteht ein regelmässiger Fährebetrieb zwischen Kowloon (Tai Kok Tsui) und dem Freihafen Shekou.

\section{Sozialökonomischer Wandel}

Die chinesische Oeffnungspolitik zeigt sich in der Bereitschaft, Auslandkapital in verschiedensten Formen $\mathrm{zu}$ akzeptieren, jedoch nur, wenn dieses den wirtschaftlichen Zielsetzungen des Landes dienlich ist. In der Freihandelszone von Shenzhen werden den ausländischen Investoren folgende Finanzierungsund Kooperationsmöglichkeiten angeboten:

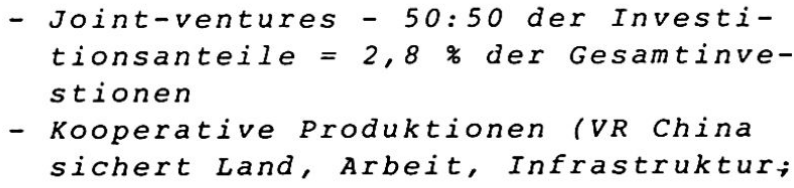


ausländischer Investor liefert Maschinenpark und sorgt für Transport bzw. Management) $=56,8 \%$ der Gesamtinvestitionen

- Kompensationshandel = 1,8 \% der Gesamtinvestionen

- Unmittelbare Verarbeitung $=38,6 \%$ der Gesamtinvestitionen

Den eigentlichen Schwerpunkt der beschleunigten Entwicklung von Shenzhen SEZ bildet der Aufbau der Leichtindustrie. Zur Zeit hat die Textilindustrie, die Elektronik und die Leichtmetallverarbeitung einen $97,5 \%$ igen Anteil an der Gesamtproduktion aller Industriebetriebe der Freihandelszone. 151 Produktionsstätten verteilen sich auf 16 verschiedene Produktionsbranchen. In diesem Initialstadium der Wirtschaftsentwicklung sind auch die marginalen 2'231 Familienbetriebe von Bedeutung, die 1984 in Heimarbeit eine Gesamtwertschöpfung von ca. SFr. 650'000.-- realisiert haben.

Die Besteuerung in der Shenzhen SEZ beträgt 15 \& und liegt unterhalb des Hongkonger Standards. Auch die vorläufig noch relativ niedrigen Bodenpreise kontrastieren mit der üblichen Praxis der Hongkonger Bodenmakler. 1984 kostete $z . B .1 \mathrm{~m}^{2}$ Land in der Shekou Industrial Zone ca. SFr. 15.--. Eine graduelle Anpassung an die Hongkonger Bodenpreise ist allerdings $\mathrm{zu}$ erwarten.

Durch Leistungslöhne versuchen die ausländischen Investoren, die allgemeine Arbeitsmoral $\mathrm{zu}$ heben und die Produktivität dem Hongkonger Niveau anzugleichen. Auf die Arbeiterschaft von Shenzhen übt diese Lohnpolitik einen grossen Anreiz aus. Die Arbeiterlöhne bewegen sich zwischen SFr. 100.-- bis 420.-- im Monat; damit liegen sie aber immer noch etwa 30 \& unter den Löhnen der Hongkonger Arbeiter.

In den Industriebetrieben der Shenzhen SEZ sind ca. 23'250 Arbeitnehmer beschäftigt, wovon nur ein Fünftel Männer sind (Gesamtbevölkerung der Shenzhen SEZ 84'057/1984). Diese Tatsache charakterisiert die lokale Beschäftigungspolitik, die oft "frühkapitalistische" Züge trägt. Das Fehlen des Streikrechts und die teilweise Kasernierung der Arbeiterschaft runden das Bild ab. Ebenso typisch ist der chronische Mangel an qualifizierten Arbeitskräften, die vor allem aus der Provinz Guangdong, aber auch aus den nördlich gelegenen Provinzen nach Shenzhen delegiert werden. Zudem kann sich aufgrund des relativ bescheidenen Lohnniveaus der Arbeiter in der Freihandelszone Shenzhen vorläufig kein Wohnungsmarkt etablieren (Abb. auf dem Titelblatt). Die von uns besichtigten provisorischen Arbeiterunterkünfte in Shekou, Shenzhen und Luohu haben einen fast "slumartigen" Charakter. Dieses Jahr wurde die erste moderne Wohnsiedlung nach westlichem Standard in Chui Chuk Yuen, in unmittelbarer Nähe des Shenzhen Reservoirs, errichtet. Für 33 Millionen Franken hat dort die Hongkonger Firma Cogi Estates Ltd. l'296 Wohnungen gebaut. Als potentielle Käufer kommen aber nur Uebersee-Chinesen und Hongkonger in Frage. Aehnliche Projekte werden in Shatouijao, Luohu und Wenjindu verwirklicht.

Für den Central Business District in Luohu wurde 1980 ein Master Plan konzipiert (Abb. 2). Einige Komplexe und Einrichtungen des zukünftigen Geschäftszentrums von Luohu/Shenzhen in der Nähe der Eisenbahnstation sind bereits in Betrieb und bieten vorläufig eine Gesamtfläche von $21^{\prime} 100 \mathrm{~m}^{2}$. Doch ein grosser Teil der angebotenen Räume ist vorläufig noch nicht vermietet. Die Unausgeglichenheit der lokalen Planung hat die Town Planning Division Hongkong zur Ueberlegung geführt, in Zukunft gemeinsam mit den Planern in Guangdong die Zonierung durchzudiskutieren (PUN 1984).

Für die Shenzhen-Planer ist Hongkong von besonderem Interesse; dies zeigen die Projekte, die fast ausschliesslich auf die Bedürfnisse der Kronkolonie ausgerichtet sind, z.B.:

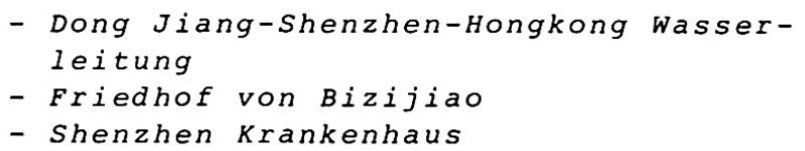

Einem grossen Wandel ist das lokale Strassennetz unterworfen. $\mathrm{zu}$ den $316 \mathrm{~km}$ Strassen von Shenzhen (WONG 1984) sind 1984 weitere $31 \mathrm{~km}$ dazugekommen. Das Hauptproblem ist im monsungeplagten Shenzhen jedoch die mindere Qualität eines grossen Teiles dieses Strassennetzes.

Eine besondere stellung innerhalb der Modernisierungspläne der Freihandelszone von Shenzhen wird dem Tourismussektor eingeräumt. An traditionellen chinesischen Feiertagen strömen Tausende von Besuchern aus der Kronkolonie nach Shenzhen; am Neujahrsfest 1985 waren es z.B. rund 290'000 Personen. Das Shenzhen Tourist Bureau hat im Jahre 1980 acht touristische Zentren und Erholungszonen geplant: Shenzhen Reservoir, Bijia Silver Lake, Xili Reservoir, Xichong Beach, Yulu Hot Springs, Lungfeng Recreation Centre, Meisha Beach, Chiwan Beach. Diese grösstenteils schon fertiggestellten Einrichtungen sollen 


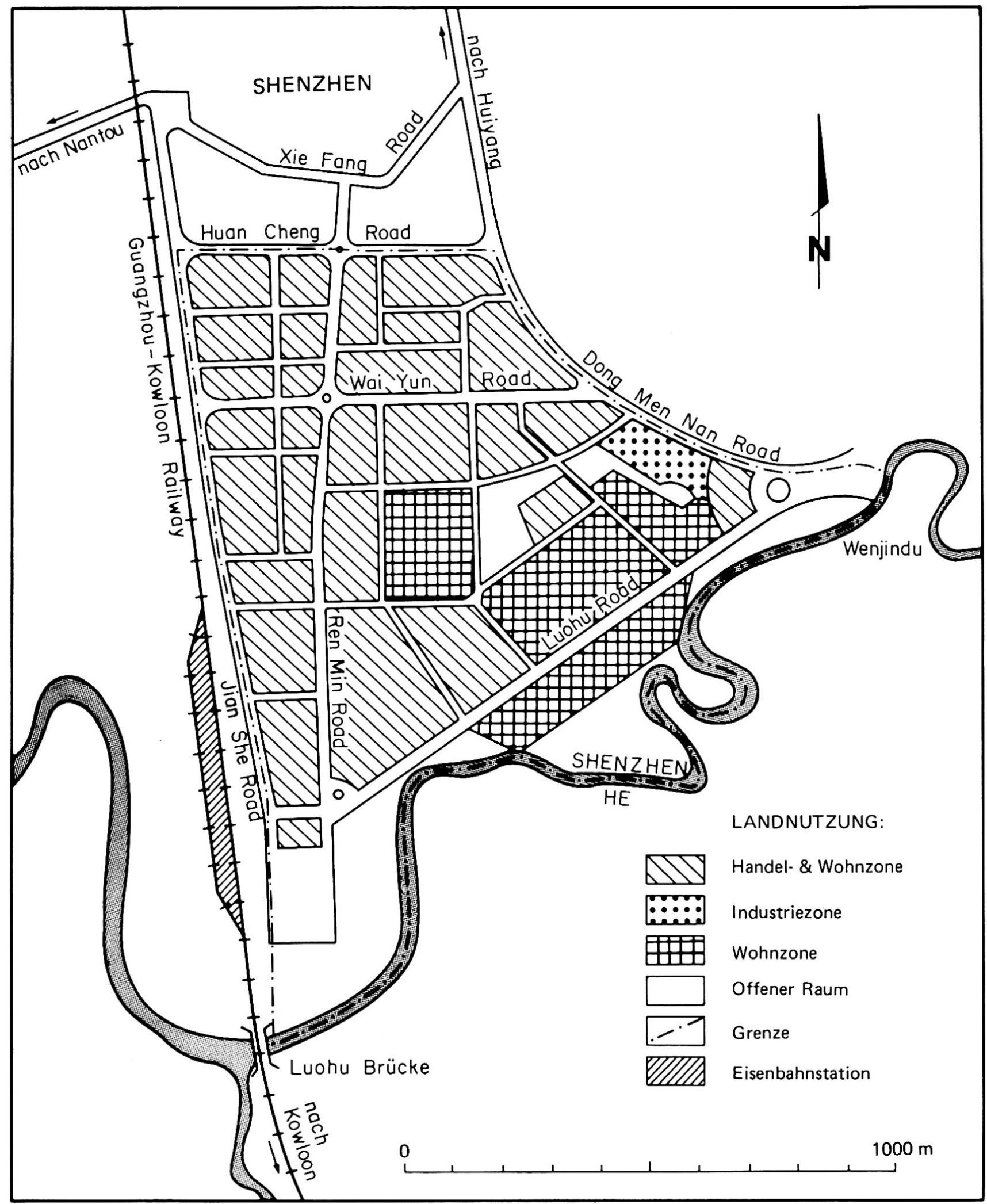

Entwurf: D. Šimko 1985

Abb. 2 Nutzungsplan von Luohu/Shenzhen 


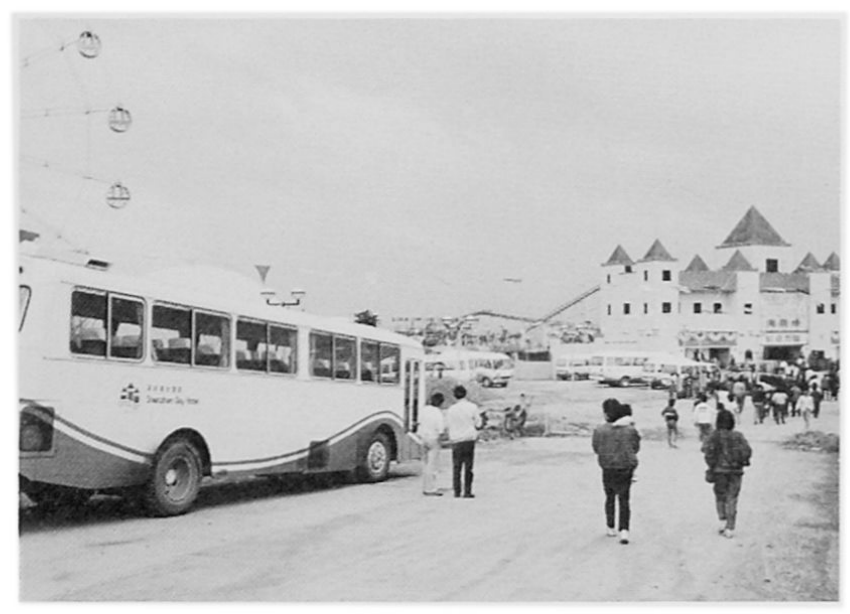

Abb. 3 Recreational Centre Xili Reservoir

täglich bis 10'000 Besucher "bewältigen" können. Der architektonische Habitus der Erholungszentren von Shenzhen ist teilweise an amerikanischen Vorbildern orientiert (Abb. 3); die Bauten weisen bereits heute starke Abnutzungserscheinungen auf.

\section{Negative Auswirkungen}

Die übereilte Industrialisierung in der Shenzhen SEZ hat die sozialen, ökonomischen und ökologischen Verhältnisse der Freihandelszone von Grund auf verändert. Dabei sind generell folgende negative Auswirkungen festzuhalten:

- Starke Verschmutzung der Flüsse im Bereich von Shenzhen, Shangbu, shahe und shekou

- Schwarzhandel, Korruption, Prostitution in den touristischen Zentren

- Demographisches Ungleichgewicht in den Industriezonen

- Beschleunigte Erosion in den neueröffneten "industrial sites"

- Wachsende Lebenskosten, wachsendes Lohngefälle zwischen lokalen und immigrierten Arbeitskräften

\section{Schlussbemerkung}

Seit 1979 versucht die VR China, einen Teil der neuen Reformpolitik in den Special Economic Zones zu verwirklichen. Die Shenzhen SEZ ist mit der Fläche von $327,5 \mathrm{~km}^{2}$ und einer Einwohnerzahl von 84'057 die grösste unter vier bestehenden SEZ. Nach dem Joint-venture-Gesetz von 1979 wurden in der Shenzhen SEZ nur 2,8 \& der Gesamtinvestitionen getätigt.
Den Löwenanteil von 56,8 \& der Gesamtinvestitionen haben noch immer die kooperativen Produktionen. Es ist dabei aber anzunehmen, dass die ganze weitere Entwicklung in Shenzhen von der politischen und ökonomischen Stabilität in der britischen Kronkolonie abhängig ist. Diese Abhängigkeit der Freihandelszone wird offenbar in Zukunft die Nutzungskosten unnötig in die Höhe treiben. Die rasante Entwicklung der Shenzhen SEZ ist dabei charakterisiert durch inkonsequente Nutzungsplanung und starken sozialen Wandel.

\section{Summary}

Since 1979 the Chinese People's Republic has attempted to implement part of the new economic policy of reform in the Special Economic Zones (SEZs). The Shenzhen SEZ is the largest of the four existing SEZs and has an area of 327,5 square kilometres and about 84'000 inhabitants. In the Shenzhen SEZ, most of the foreign investment is concentrated in the real estate/construction business and tourism and not exclusively in manufacturing. The joint-ventures have proved to be disappointing and currently account for only 2,8 of of the total investment. On the other hand, cooperative production is still the principal form of investment with 56,8 \& of the total. The economic and technological dependence of Shenzhen SEZ on Hongkong is likely to have an adverse effect on production costs and the price of land. The accelerated development in Shenzhen SEZ has been accompanied by inadequate infrastructure provision and serious social problems.

\section{Literatur}

THE SOUTH CHINA MORNING POST, JUIY 3, 1985

INTERNATIUNAL HERALD TRIBUNE, JUIY 6/7, 1985

KOSTA, J. (1985): Die Wirtschaftsreform in der VR China. In: Listy, Jg. 15, 2, S. 27-32, Rom.

PUN, K.S. (1984): Urban Planning in Hong Kong. In: Third World Planning Review, Vol. 6, No 1 .

WONG, K.Y./CHU, K.Y. (1984): Export Processing Zones and Special Economic Zones as Generators of Economic Development: The Asian Experience. In: Geografiska Annaler, 66 B.1.

WONG, K.Y. (1982): Shenzhen Special Economic Zone, Hongkong. 